\title{
Implementation and Demonstration of Receiver- Coordinated Distributed Transmit Beamforming across an Ad-hoc Radio Network
}

\author{
Patrick Bidigare, Miguel Oyarzyn, David Raeman, Dan Chang, Dave \\ Cousins, Rich O'Donnell Charlie Obranovich \\ Raytheon BBN Technologies Arlington, VA 22209 \\ [pbidigare, moyarzun, draeman, dchang, dcousins, rodonnell, \\ cobranovich]@bbn.com
}

\author{
D. Richard Brown III \\ ECE Department \\ Worcester Polytechnic Institute \\ Worcester, MA 01609 \\ drb@wpi.edu
}

\begin{abstract}
Distributed transmit beamforming using an ad-hoc network of $10 \mathrm{RF}$ transmitters was demonstrated using radio nodes developed from off-the-shelf components and modules. A time-slotted protocol allowed carrier phases from each transmitter to be measured at a receiver and fed back to the transmitters where Kalman filters were used to predict the offset phases and frequencies. Offsets were digitally compensated for during beamforming intervals. Beamforming gain within $0.1 \mathrm{~dB}$ of ideal was demonstrated across $1 \mathrm{~km}$ at $910 \mathrm{MHz}$. This is the first report (to our knowledge) of a successful outdoor RF distributed transmit beamforming experiment using independent clocks at this scale.
\end{abstract}

\section{INTRODUCTION}

Transmit beamforming is a physical-layer communication technique in which an information source transmits a radio frequency signal over two or more antennas and aligns the phases of the transmissions across the antennas such that, after propagation, the signals combine constructively at the intended destination. The advantages of transmit beamforming are welldocumented: increased power on target, increased range, increased data rate, and/or potentially improved security and interference reduction since transmissions are directed and less transmit power is scattered in unintended directions.

Researchers have recently begun to consider the possibility of distributed transmit beamforming in which two or more separate information sources simultaneously transmit a common message and control the phase of their transmissions so that the signals constructively combine at an intended destination. Distributed transmit beamforming, sometimes also called "collaborative beamforming", is a powerful technique that offers the potential gains of conventional antenna arrays to wireless communication systems composed of multiple singleantenna transmitters with independent local clocks.

Several techniques have been proposed to enable distributed transmit beamforming including receivercoordinated one-bit feedback [1-3], receiver-coordinated explicit-feedback [4-5], master-slave synchronization with retrodirective transmission [6], round-trip [7-8], and two-way synchronization with retrodirective transmission [9-10]. Distributed transmit beamforming has also been considered for the downlink of cellular networks under the title "coordinated multipoint" (CoMP), e.g. [11], which uses a receivedcoordinated full-feedback approach similar to [4-5]. Each of these techniques has advantages and disadvantages in particular applications, as discussed in the survey article [12].

While the focus of the prior work has been primarily theoretical, several recent papers have considered practical implementation issues and reported laboratory-based experimental studies. A practical DSP-centric approach to distributed transmit beamforming was described in [13]. Experimental studies have been reported recently in [14-17]. This paper reports on an experimental study of receivercoordinated explicit-feedback distributed transmit beamforming with 10 nodes transmitting at $900 \mathrm{MHz}$ over a $1 \mathrm{~km}$ outdoor wireless link. To the best of the authors' knowledge, this is the first reported study of large-scale distributed transmit beamforming in an outdoor environment.

Distributed transmit beamforming is the key critical enabling technology for achieving distributed coherent communications across a flat fading channel using an ad-hoc network of radio transmitters, as shown in Fig. 1 .

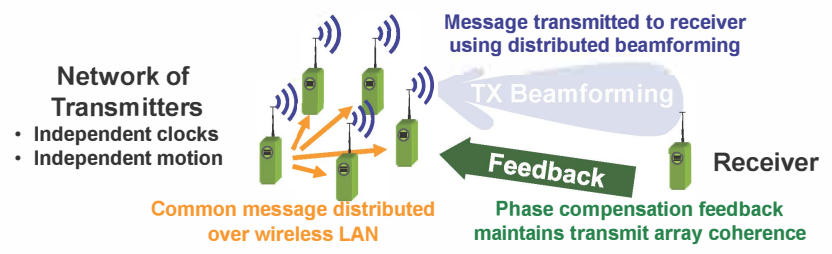

Fig. 1 Receiver-coordinated distributed transmit beamforming allows an adhoc network of transmitters to achieve longer communication ranges or higher data rates.

The power at the receiver than can be generated grows, on average, with the square of the number of coherent transmitters. This allows network of low-power radios to be used in place of a single high-power or high-gain radio. It can enable considerably longer communication ranges or higher data rates that are possible with a single radio. 
The fundamental challenge in achieving distributed transmit beamforming is that independent clock drifts, along with independent transmitter motions produce highly nonstationary channels. Feedback latency from the receiver is generally much longer than the period over which the channel remains constant, and thus cannot be addressed using the conventional block-stationarity approximations. The approach we take in this paper is to model the carrier phase offsets from the transmitters to the receiver as stochastic random processes. We track these offsets using Kalman filters running on the transmitters which are updated periodically with phase offset measurements from the receiver. In section II we provide a mathematical summary of our approach. Section III discusses our practical implementation of this technique on testbed radio hardware. The highlight of this paper is in section IV where the results of our over-the-air experiments are summarized. Section V concludes with future work based on the demonstrated technique.

\section{TECHNIQUE}

\section{A. Description}

Our Kalman filter (KF) based technique uses a time-slotted forward channel for channel sounding and transmit beamforming along with carrier offset measurements sent from the receiver to the transmitters over a feedback channel, as illustrated in Fig. 2 .



Fig. 2 Our beamforming approach uses short channel sounding slots and long transmit beamforming slots on the forward link along with sounding feedback on the reverse link.

The technique proceeds according to the following steps:

1. Receiver initially establishes timeslots.

2. Each transmitter is assigned its own short sounding timeslot (red, allareen), followed by a long common beamforming slot (purple)

3. Each transmitter broadcasts its uncompensated carrier during the sounding slot.

4. The receiver determine the carrier phase offset of each of the transmitters

5. These phase offsets are broadcast back to the transmitters over a feedback channel (blue)

6. Each transmitter uses a Kalman filter (KF) to track its carrier phase and frequency relative to the receiver. The measured offset forms a measurement update

7. The KF is used to predict the initial phase and frequency offset at the start of the beamforming slot

8. Each transmitter applies a digital phase and frequency correction to its baseband samples while beamforming

9. Steps $3-7$ are repeated

\section{B. Kalman Filter Formulation}

A fairly extensive description and mathematical formulation of the technique can be found in [5] and [13]. In this paper we concentrate on our practical implementation in testbed hardware. We implemented, on each transmitter, a Kalman filter to track the phase and frequency offset between that transmitter's carrier and the carrier of the receiver. The filter tracks the state space

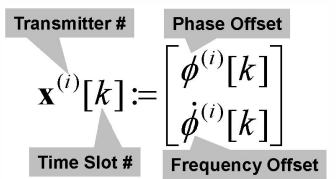

which evolves according to

$$
\mathbf{x}^{(i)}[k+1]=\left[\begin{array}{cc}
1 & \Delta t \\
0 & 1
\end{array}\right] \mathbf{x}^{(i)}[k]+\mathbf{v}^{(i)}[k]
$$

During the $k^{\text {th }}$ sounding slot for transmitter $i$, the receiver makes a (noisy) measurement of the carrier phase offset

$$
\begin{gathered}
\text { Carrier Phase } \\
\text { Offset Measurement } \\
\hat{\phi}^{(i)}[k]=\left[\begin{array}{ll}
1 & 0
\end{array}\right] \mathbf{x}^{(i)}[k]+w^{(i)}[k]
\end{gathered}
$$

which is fed back to the transmitter where it is used to update the filter estimate of the state. The Kalman filter uses a twoparameter process noise covariance model of the form

$$
E\left[\left(\mathbf{v}^{(i)}\right)^{T} \mathbf{v}^{(i)}\right]=q_{11}\left[\begin{array}{cc}
\Delta t & 0 \\
0 & 0
\end{array}\right]+q_{22}\left[\begin{array}{cc}
\Delta t^{3} / 3 & \Delta t^{2} / 2 \\
\Delta t^{2} / 2 & \Delta t
\end{array}\right]
$$

where $q_{11}$ and $q_{22}$ are diffusion and white-noise acceleration coefficients, obtained from oscillator specifications and measured motion data, as discussed below.

An efficient estimator of phase (a matched filter in white noise) is assumed, thus the measurement noise variance is given by the Cramer-Rao bound

$$
E\left[|w[k]|^{2}\right] \approx \frac{1}{2 S N R}
$$

The integrated SNR is data-adaptively determined from quiescent and signal-bearing samples.

\section{Clock and Motion Induced Process Noise}

As discussed in [18], a two-state model is generally sufficient for capturing dynamics of stochastic clock drift. Parameters $q_{11}^{\text {clock }}, q_{22}$ cock can be determined from oscillator Allan deviation specifications. Fig. 3 shows example clock process noise realizations for the Rakon ovenized clocks used in our testbed radios. Over short timescales, the clock phase process noise is dominated by the Brownian motion component $q_{11} \Delta t$ with variance growing linearly with time. 


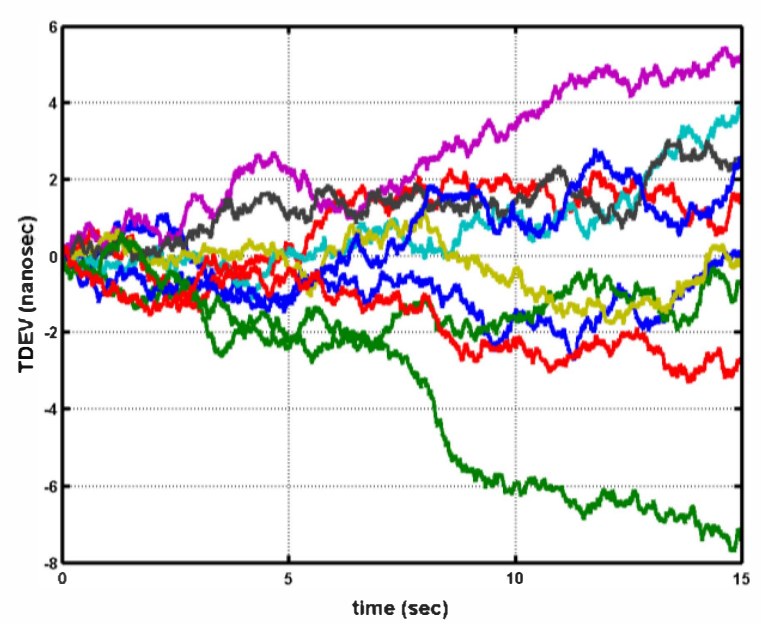

Fig. 3 Example Clock Dynamics

The number of states needed to model motion depends on the application. To keep the motion model simple and parsimonious with the clock model, we considered the twostate Kalman filter described above. The corresponding evolution equation corresponds to a "white noise acceleration" model [19]. Parameters $q_{11}^{\text {motion }}, q_{22}$ motion were determined using motion measurements taken from a tethered aerostat platform, instrumented with both INS and GPS sensors. Data collected over a 1 hour period is shown in Fig. 4 .

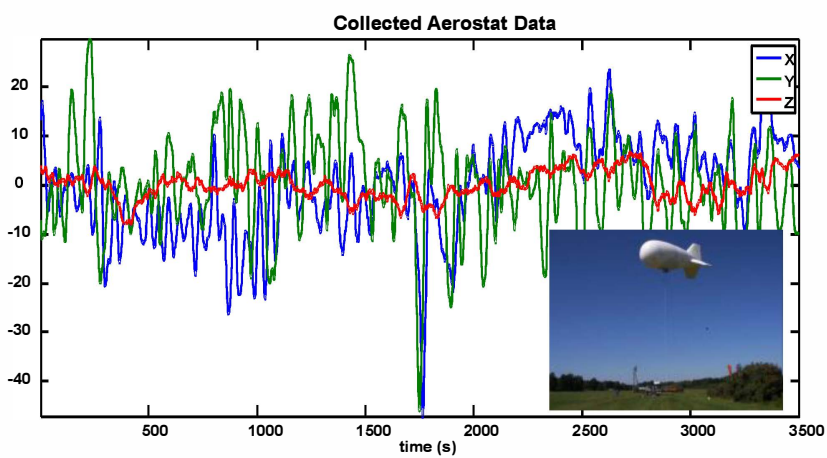

Fig. 4 Both inertial and GPS measurements were taken using an instrumentation package mounted on a tethered lighter-than-air platform Collected data was used to derive motion-induced process noise parameters.

Motion and clock parameters are independent, so process noise variances add:

$$
q_{11}=q_{11}^{\text {clock }}+q_{11}^{\text {motion }} \quad q_{22}=q_{22}^{\text {clock }}+q_{22}^{\text {motion }}
$$

\section{IMPLEMENTATION}

\section{A. Hardware}

This technique was implemented on a developmental radio platform shown in Fig. 5. The testbed hardware was constructed entirely of off-the-shelf modules or components without needing any exotic clocks or external timing sources. The carrier phase estimation and tracking were implemented on an embedded digital signal processor, and high-speed baseband phase and frequency compensation were implemented on a low-power FPGA. A custom tunable transceiver was used for channel sounding and beamforming. The system utilized an oven-controlled crystal oscillator (OCXO) reference clock to provide frequency stability between sounding intervals. An 802.11 WiFi link was used as the feedback channel to pass phase estimates from the receiver to the transmitters during channel sounding intervals.

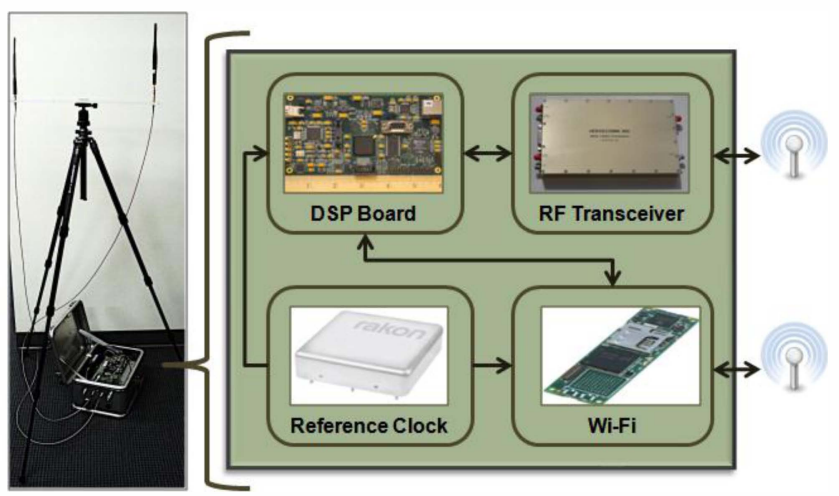

Fig. 5 Modular testbed radio provides forward and reverse links needed for experiment along with digital signal processing capabilities.

\section{B. Processing}

The noisy phase measurements are fed back from the receiver to the transmitters where they are used as measurement updates to Kalman filters with state spaces described above. The phase measurement noise at the receiver is determined by measuring the phase noise during a quiescent timeslot. The Kalman filter is used to predict transmitter $i$ 's carrier phase and frequency $\hat{\mathbf{x}}^{(i)}[k \mid k]$ at the start of the transmit beamforming sub-slot. The carrier phase and frequency are digitally pre-compensated at baseband. The baseband beamformed signal seen at the receiver during timeslot $k$ is then

$$
\left.\sum_{i=1}^{M} A^{(i)}[k] \exp (j(\underbrace{\left(\phi^{(i)}[k]+(t-t[k]) \dot{\phi}^{(i)}[k]\right.}_{\text {True Phase }})-\underbrace{\left(\hat{\phi}^{(i)}[k \mid k]+(t-t[k]) \hat{\phi}^{(i)}[k \mid k]\right)}_{\text {Predicted Phase }})\right)
$$

where $A^{(i)}[k]$ is the (approximately constant) amplitude of the $i^{\text {th }}$ transmitter as seen by the receiver in the $k^{\text {th }}$ timeslot.

\section{RESULTS}

\section{A. Experimental Setup}

Experimental over-the-air tests were conducted in the Fall 2011 at Whitetail Ski Resort in Mercersburg, PA. Fig. 6 shows a typical experimental setup.

A sparse array of 10 fully independent transmitter nodes was distributed over $\sim 30$ meters at the top of a ski slope. The receive node was placed line-of-sight at a standoff of 1 kilometer. A high-gain antenna was used to close the 802.11 WiFi link used for the feedback channel. 


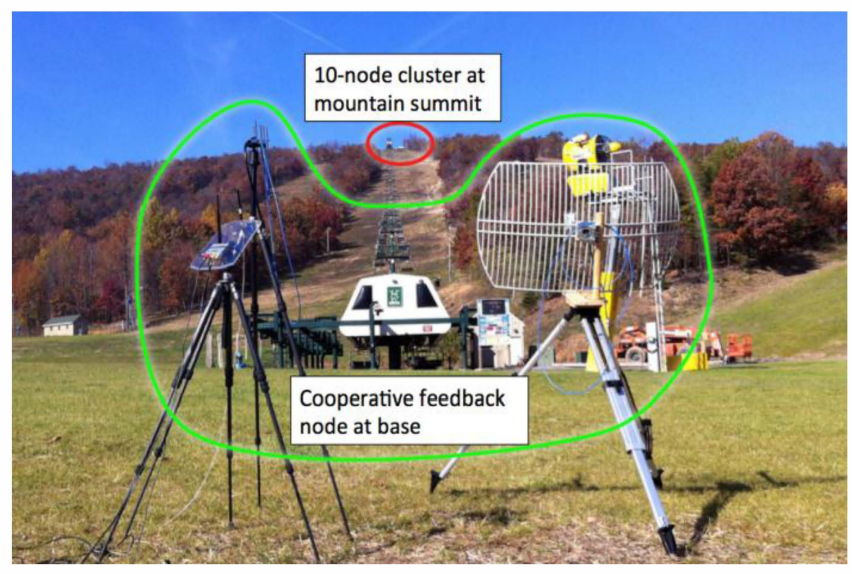

Fig. 6 Experimental setup of distributed transmit beamforming

The non-stationary outdoor environment included many real-world RF challenges such as pulsed in-band interference, multipath (including metal poles along the direct propagation path), and motion in the immediate test area (although not by the participating transmit nodes). The technique was successfully demonstrated on numerous occasions between September and November 2011 in similar outdoor experiments.

TABLE I. EXPERIMENT PARAMETERS

\begin{tabular}{lcclc} 
Parameter & Value & & Parameter & Value \\
\cline { 1 - 2 } \cline { 5 - 6 } Antenna height & $2 \mathrm{~m}$ & & Center frequency & $910 \mathrm{MHz}$ \\
Standoff range & $1 \mathrm{~km}$ & & Waveform & $\mathrm{CW}$ \\
Transmit power & $0.5 \mathrm{~W}$ & & Sounding duration & $100 \mathrm{us}$ \\
Transmitters & 10 & & Sounding frequency & $5 \mathrm{~Hz}$ \\
Network radio & $\mathrm{WiFi}$ & &
\end{tabular}

\section{B. Distributed Transmit Beamforming Performance}

Individual transmitter amplitudes were measured during sounding slots and aggregate beamformed power was measured during the beamforming slot and displayed using a GUI connected to the receive node. Fig. 7 shows a screen capture of the GUI during an experiment with time progressing on the $\mathrm{x}$-axis and measured receive power on the $\mathrm{y}$-axis.

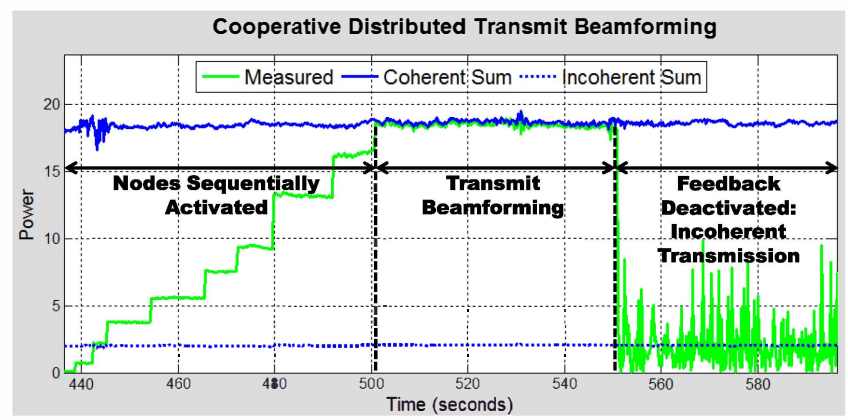

Fig. 7 Real-time graphical user interface display shows optimal coherent (blue solid), incoherent (blue dashed) and measured (green) power at the receiver during the experiment.
The left-most segment of Fig. 7 shows each of the 10 transmitters being sequentially activated. In the middle segment, the full array is maintaining coherence within $0.1 \mathrm{~dB}$ of the ideal beamforming gain. The measured power is compared against the ideal coherent power, which is calculated by first summing and then squaring the amplitudes of the signals received by the individual transmitters. The right segment shows the array quickly becoming incoherent when the phase estimation feedback is disabled.

The authors believe that this is the first over-the-air receiver-coordinated distributed transmit beamforming to be demonstrated at this scale using radio frequencies. The implementation and test show that near-optimal beamforming can be achieved when feedback latency is sufficiently small to allow accurate Kalman filter prediction of the transmitter phases.

\section{CONCLUSIONS}

Our implementation of this receiver-coordinated transmit beamforming technique was an important first-step towards realizing the potential of distributed coherent communications from a wireless network of radios to a distant receiver. We were able to verify the analyses in [5] showing that nearoptimal beamforming could be achieved with off-the-shelf electronics, operating at significant ranges with real-world channel impairments.

The transmit beamforming demonstration was done using exclusively $\mathrm{CW}$ tones. A future step will incorporate the time alignment of the a common baseband communications signal across the distributed transmit network to allow coherent distributed communications to be demonstrated over singlepath channels.

The transmit node mobility in our experiments was limited by the latency of a commercial WiFi feedback link. A future step will more tightly integrate the forward link radio with the feedback radio to greatly reduce the feedback latency and allow update rates of 100 to $200 \mathrm{~Hz}$. This is expected to allow transmit coherency to be maintained for both pedestrian and vehicular motion.

Finally, to operate in the multipath environments, the delayonly channel model is insufficient and the technique must be extended to allow transmit precoding (baseband filtering) in addition to phase and frequency compensation.

\section{REFERENCES}

[1] R. Mudumbai, J. Hespanha, U. Madhow, and G. Barriac, "Scalable feedback control for distributed beamforming in sensor networks," in IEEE International Symp. on Information Theory (ISIT), Adelaide, Australia, September 2005, pp. 137-141

[2] R. Mudumbai, B. Wild, U. Madhow, and K. Ramchandran, "Distributed beamforming using 1 bit feedback: from concept to realization," in 44th Allerton Conf. on Comm, Control, and Computing, Monticello, IL, Sep. 2006, pp. $1020-1027$.

[3] R.Mudumbai,J.Hespanha,U.Madhow, andG.Barriac, "Distributed transmit beamforming using feedback control," IEEE Trans. on Information Theory, vol. 56, no. 1, pp. 411-426, January 2010

[4] Y.S. Tu and G.J. Pottie, "Coherent cooperative transmission from multiple adjacent antennas to a distant stationary antenna through 
AWGN channels," in IEEE Vehicular Technology Conf. (VTC), Birmingham, AL, Spring 2002, vol. 1, pp. 130-134.

[5] D. R. Brown, P. Bidigare, and U. Madhow, "Receiver coordinated distributed transmit beamforming with kinematic tracking," in IEEE International Conference on Acoustics, Speech, and Signal Processing (ICASSP 2012), Kyoto, Japan, March 2012.

[6] R. Mudumbai, G. Barriac, and U. Madhow, "On the feasibility of distributed beamforming in wireless networks," IEEE Trans. on Wireless Communications, vol. 6, no. 5, pp. 1754-1763, May 2007

[7] Ipek Ozil and D.R. Brown III, "Time-slotted roundtrip carrier synchronization," in Proceedings of the 41st Asilomar Conference on Signals, Systems, and Computers, Pacific Grove, CA, November 4-7, 2007, pp. $1781-1785$

[8] D.R. Brown III and H.V. Poor, "Time-slotted round-trip carrier synchronization for distributed beamforming," IEEE Trans. on Signal Processing, vol. 56, no. 11, pp. 5630-5643, November 2008.

[9] R.D. Preuss and D.R. Brown, "Retrodirective distributed transmit beamforming with two-way source synchronization," in Information Sciences and Systems (CISS), 2010 44th Annual Conference on, march 2010, pp. $1-6$.

[10] R. Preuss and D.R. Brown III, "Two-way synchronization for coordinated multi-cell retrodirective downlink beamforming," IEEE Trans. on Signal Processing, November 2011.

[11] R. Irmer, H. Droste, P. Marsch, M. Grieger, G. Fettweis, S. Brueck, H.P. Mayer, L. Thiele, and V. Jungnickel, "Coordinated multipoint: Concepts, performance, and field trial results," Communications Magazine, IEEE, vol. 49, no. 2, pp. 102-111, February 2011.

[12] R. Mudumbai, D.R. Brown III, U. Madhow, and H.V. Poor, "Distributed transmit beamforming: Challenges and recent progress," IEEE Communications Magazine, vol. 47, no. 2, pp. 102-110, February 2009.
[13] Mudumbai, Raghu; Madhow, Upamanyu; Brown, Rick; Bidigare, Patrick; , "DSP-centric algorithms for distributed transmit beamforming," Signals, Systems and Computers (ASILOMAR), 2011 Conference Record of the Forty Fifth Asilomar Conference on , vol., no., pp.93-98, 6-9 Nov. 2011

[14] D.R. Brown, Boyang Zhang, B. Svirchuk, and Min Ni, "An experimental study of acoustic distributed beamforming using round-trip carrier synchronization," in Phased Array Systems and Technology (ARRAY), 2010 IEEE International Symposium on, oct. 2010, pp. 316 323.

[15] M. M. U. Rahman, H. E. Baidoo-Williams, S. Dasgupta, and R. Mudumbai, "Fully wireless implementation of distributed beamforming on a software-defined radio platform," in the 11th ACM/IEEE Conference on Information Processing in Sensor Networks (IPSN), 2012.

[16] F. Quitin, M. M. U. Rahman, R. Mudumbai, and U. Madhow, "Distributed beamforming with software-defined radios: frequency synchronization and digital feedback," in IEEE Globecom 2012, December 2012. to appear.

[17] F. Quitin, U. Madhow, M. Rahman, and R. Mudumbai, "Demonstrating distributed transmit beamforming with software-defined radios," in World of Wireless, Mobile and Multimedia Networks (WoWMoM), 2012 IEEE International Symposium on a, pp. 1-3, june 2012.

[18] Lorenzo Galleani, "A tutorial on the two-state model of the atomic clock noise,” Metrologia, vol. 45, no. 6, pp. S175-S182, December 2008.

[19] Yaakov Bar-Shalom, X. Rong Li, and Thiagalingam Kirubarajan, Estimation with Applications to Tracking and Navigation, John Wiley and Sons, 2001 